\title{
Structure-based pharmacophore models generation and Combinatorial Screening of ICE Inhibitors
}

\author{
Zhang Yanling ${ }^{\mathrm{a}}$, Wang Yuanming, QiaoYanjiang ${ }^{\mathrm{b}}$ \\ School of Chinese Pharmacy, Beijing University of Chinese Medicine \\ Beijing 100102, China \\ Email: ${ }^{a}$ zhangyanling@bucm.edu.cn, ${ }^{b}$ yjqiao@bucm.edu.cn
}

Correspondence: Prof. Yanjiang Qiao, School of Chinese Pharmacy, Beijing University of Chinese Medicine, No. 6 Wangjing Zhong Huan Nan Lu, Chaoyang District, Beijing 100102, China. E-mail: yjqiao@263.net Phone: +86 1084738661 Fax:+86

1084738611

\begin{abstract}
Eight structure-based pharmacophore models of Interleukin-1 $\beta$ converting enzyme (ICE) inhibitors were generated by LigandScout based on ICE inhibitor complexes from the Protein Data Bank (PDB). A combinatorial screening method based on multiple pharmacophore models were proposed in present study, since the binding pockets of different complexes were different, the structure-based pharmacophore models have a high specificity and cannot cover all the active molecules. Based on the screening results of MDDR and the metrics of $E$ and $A \%$, a new metric $C A I$ (comprehensive appraisal index) was defined and used to determine the threshold of hit frequency of molecules which screened by the combinatorial screening method. According to the threshold, the potential ICE inhibitors were then identified from traditional Chinese medicine with the method of combinatorial screening with eight models. The molecules hit by not less than five pharmacophore models were taken as the screening objects of ICE inhibitor, and 781 molecules were obtained.
\end{abstract}

Keywords- Interleukin-1及 converting enzyme; structure-based pharmacophore; combinatorial screening of multiple pharmacophores; comprehensive appraisal index; Identification from Chinese Herbs

\section{INTRODUCTION}

Chinese herbs, which was concerned with low toxicity and side-effects, are an important source for modern drug discovery. Virtual screening by pharmacophore was discussed as a useful approach to get successful compounds from Chinese herbs ${ }^{[1]}$. In order to identify potential compounds with Interleukin- $1 \beta$ converting enzyme (ICE) inhibitor activity, the structure-based pharmacophore models were generated by LigandScout software in present study.

The receptor-ligand complexes were used to construct structure-based pharmacophore models by interpret the interaction between the receptor and ligand. The chemical characteristics of complementarity revealed by the interaction are taken into account into the structure-based models, as well as the space complementary between the receptor and the ligand. However, since the binding pocket is flexible, the models from different complexes with different classes of ligands can only reflect limited binding mode. Therefore, the screening by a single structure-based pharmacophore model cannot cover all the active molecules with various skeleton types, which will cause a high false positive rate. A combinatorial screening method was proposed in present study, which mean the compounds hit by different models are the most likely active compounds. By this method, each compounds in the database should be matched by each model, which was generated from every complex.

IL-1 precursor can be converted to the activated cytokine IL- $1 \beta$ by ICE, and IL- $1 \beta$ is associated with a variety of inflammatory diseases, such as viral infections, tumors, rheumatic diseases ${ }^{[2,3]}$. ICE has become an important target for the treatment of inflammatory diseases. So, it's significant to find potential ICE inhibitors from Chinese herbs. 781 compounds were hit by combinatorial screening method and the ICE inhibitory of some hit compounds was verified by literatures.

\section{DATA AND METHOD}

\section{A. Pharmacophore model generation}

Eight crystal structures of human ICE were obtained from the Protein Data Bank (PDB) database (Table I), which were analyzed by $\mathrm{x}$-ray diffraction method with a resolution of less than $3 \AA$. The structures were preprocessed and used for the generation of pharmacophore models. The crucial interaction chemical characteristics and space complementary between the protein and ligands were then detected and interpreted by LigandScout software to create and visualize advanced 3D pharmacophore models.

\begin{tabular}{|c|c|c|c|}
\hline PDB_ID & Resolution( $(\AA)$ & Ligand_ID & Ligand action (nM) \\
\hline 1RWK & 2.30 & 158 & - \\
\hline 1RWM & 2.70 & Q2Y & $\mathrm{Ki}=120$ \\
\hline $1 \mathrm{RWN}$ & 2.00 & 4QB & - \\
\hline 1RWO & 2.10 & BTH & - \\
\hline 1RWP & 2.20 & HQC & - \\
\hline $1 \mathrm{RWW}$ & 2.80 & OQB & $\operatorname{IC} 50=5$ \\
\hline $1 \mathrm{RWX}$ & 1.85 & YBH & $\mathrm{Ki}=43$ \\
\hline $1 \mathrm{BMQ}$ & 2.50 & MNO & IC $50=38$ \\
\hline
\end{tabular}

Since the pharmacophore models constructed by LigandScout have multiple features in the same position, the models cannot be used for virtual screening directly. In the present study, the pharmacophore models were then 
adjusted and converted into Catalyst 4.11 for virtual screening. The adjustment method was used to simplify the models by the critical features, which were measured and chosen by the specific priority and the interaction distance between the amino acid residues and the ligand. The simplified models were called sub-pharmacophore in this paper.

A number of metrics were proposed for models evaluation, such as percent yield of actives (Y\%), enrichment factor $(\mathrm{E})$, percent ratio of the actives in the hit list $(\mathrm{A} \%)$, and goodness of hit list $(\mathrm{GH}){ }^{[4,5]}$. Of these, $\mathrm{E}$ and $\mathrm{A} \%$ are two of the most widely used and the simpler measures, which are described by the following equation: $\mathrm{A} \%=(\mathrm{Ha} / \mathrm{A}) \times 100, \mathrm{E}=(\mathrm{Ha} / \mathrm{Ht}) /(\mathrm{A} / \mathrm{D})$, where $\mathrm{D}$ is the number of compounds in the database, $\mathrm{A}$ is the number of active compounds in the database, $\mathrm{Ht}$ is the number of compounds in the hit list, and $\mathrm{Ha}$ is the number of active compounds in the hit list. The higher $\mathrm{A} \%$ value mean the more coverage of active compounds in the hit list. Because different classes of active compounds may bind in different modes, more hit compounds needed to obtain more active compounds. Therefore, the higher A\% value may be obtained only by compromising the selectivity of the pharmacophore models. $\mathrm{E}$ value is used to evaluate the ability of the models to identify active compounds from the non-active compounds. A good model should have the two abilities that can cover more active compounds and can enrich the active compounds in hit list. In present study, a metric CAI value (Comprehensive Appraisal Index) was proposed to take into account both the accuracy and efficiency of the models. The definition of CAI is described by the equation as follows: $\mathrm{CAI}=\mathrm{E} \times \mathrm{A} \%$. In present study, MDDR (MDL Drug Data Report: Version 2007.2) was used as the testing database to evaluate the pharmacophore models, and the higher CAI value is considered to be the better model.

\section{B.Combinatorial screening of multiple pharmacophores}

According to the combinatorial screening method, the hit lists of all 8 pharmacophore models were integrated to obtain the hit frequency of each compounds. Different with the evaluation of pharmacophore models, the CAI value was used to evaluate the proper hit frequency for the virtual screening. For combinatorial screening, the metrics were adjusted to the equation as follows: $\Sigma \mathrm{A} \%=\Sigma \mathrm{Ha} / \mathrm{A} \times 100 \%$, $\Sigma \mathrm{E}=(\Sigma \mathrm{Ha} / \Sigma \mathrm{Ht}) /(\mathrm{A} / \mathrm{D}), \mathrm{CAI}=\Sigma \mathrm{E} \times \Sigma \mathrm{A} \%$, where the $\Sigma \mathrm{Ht}$ is the number of compounds in the hit list that contained the compounds matched greater than or equal to the frequency times, and the $\Sigma \mathrm{Ha}$ is the number of active molecules in the hit list which contained the compounds matched greater than or equal to the frequency times. For an example, the hit frequency is 5 mean all the hit compounds can match 5 different models. And if for 5 hit frequency, a hit list is obtained with 4 hit compounds, which contains 2 active compounds, then the $\Sigma \mathrm{Ht}$ is 4 , and the $\Sigma \mathrm{Ha}$ is 2 . For a single molecule, if which can match more models, will be better fit the binding pocket of the receptor. For a better hit frequency of the combinatorial screening, the $\Sigma \mathrm{E}$ should be greater than 1 to ensure the enrichment of the screening, and the $\Sigma \mathrm{A} \%, \Sigma \mathrm{E}$ and $\Sigma \mathrm{CAI}$ should all be greater than the best structure-based pharmacophore model. After the best proper frequency was defined, it will be used as a threshold for the combinatorial screening of potential compounds from Chinese herbs.

\section{In silico screening}

Virtual screening was performed with the Catalyst software and using the FAST flexible searching technique. The MDDR database, which contains 177981 compounds and 972 compounds clearly marked with ICE inhibition activity, was used as the test database. The resulting hit molecule numbers were used to calculate the $\mathrm{E}, \mathrm{A} \%$, CAI, $\Sigma \mathrm{E}, \Sigma \mathrm{A} \%$ and $\Sigma \mathrm{CAI}$ value, and ranked for the best model and the threshold of hit frequency. Then the combinatorial screening method will be performed to screen the TCMD database which contains 23033 compounds from the Chinese herbs.

\section{RESULTS AND DISCUSSION}

The pharmacophore models were automatically generated by the LigandScout software, and included the features like: hydrogen bond donor (D), Hydrogen bond acceptor (A), Hydrophobic groups (H), Ring Aromatic groups (R) and Negative groups $(\mathrm{N})$ (shown in TableII). Several excluded volumes were also generated in the models to show the space complementary. All the 8 models contain hydrogen bond acceptors, hydrogen bond donors and hydrophobic groups, indicating that these features are key features of the ICE inhibitors.

The sub-pharmacophore was obtained by analyzing the critical features of each complex (the complex with the PDB ID 1BMQ was taken as an example shown in Fig. 1, which showed the structure-based pharmacophore in the active pocked detected by LigandScout and the sub-pharmacophore of the complex). The evaluation metrics was obtained by the virtual screening of the MDDR database (shown in Table III). The sub-pharmacophore from the 1RWK complex was the best model which has the highest CAI value, and was used as a reference to determine the proper hit frequency of the combinatorial screening.

TABLE II.ICE INHIBITOR PHARMACOPHORE MODELS CONSTRUCTED BY

\begin{tabular}{cccc}
\multicolumn{4}{c}{ PDB COMPLEXES } \\
\hline PDB_ID & Features & PDB_ID & Features \\
\hline 1BMQ & HAAAADARAH & $1 \mathrm{RWO}$ & ADHAAAHNDAARA \\
1RWK & AAADAAAANA & $1 \mathrm{RWP}$ & DHARAAANAAAH \\
$1 \mathrm{RWN}$ & NAAHHDAAADA & $1 \mathrm{RWM}$ & AAHAHNRAAADA \\
$1 \mathrm{RWW}$ & DDHNAHAAAA & $1 \mathrm{RWX}$ & AAHAAAAHRDDAAHN \\
\hline
\end{tabular}




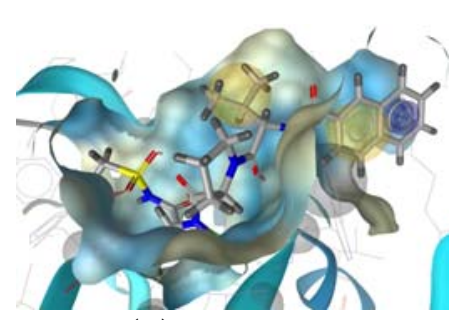

( a)

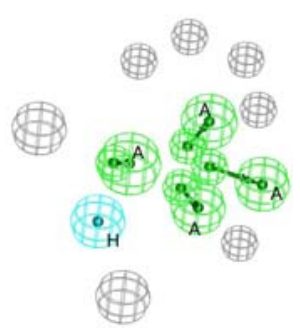

(b)
Figure 1. Pharmacophore model of 1BMQ complex

(a)structure-based pharmacophore in the active pocked detected by LigandScout;(b) the sub-pharmacophore of the complex.

TABLE III. ICE INHIBITOR SUB-PHARMACOPHORE MODELS AND RESULTS OF VIRTUAL SCREENING

\begin{tabular}{ccccccc}
\hline ID & Features & Ha & Ht & $\mathbf{A \%}$ & $\mathbf{E}$ & $\mathbf{C A I}$ \\
\hline 1bmq & HAAAA & 171 & 17535 & 41.61 & 4.22 & 1.76 \\
1rwk & ADAAA & 139 & 5841 & 33.82 & 10.31 & 3.49 \\
1rwm & HAAA & 243 & 41146 & 59.12 & 2.56 & 1.51 \\
1rwn & AHAAA & 160 & 8614 & 38.93 & 8.04 & 3.13 \\
1rwo & AAHDA & 106 & 6717 & 25.79 & 6.83 & 1.76 \\
1rwp & HAAA & 229 & 53216 & 55.72 & 1.86 & 1.04 \\
1rww & DHAAA & 159 & 10000 & 38.69 & 6.89 & 2.66 \\
1rwx & AHRAH & 113 & 6505 & 27.49 & 7.52 & 2.07
\end{tabular}

The combinatorial screening results of MDDR database were shown in Table IV. Compared with the best sub-pharmacophore model $1 \mathrm{RWK}$, the $\Sigma \mathrm{A} \%, \Sigma \mathrm{E}$ and $\Sigma \mathrm{CAI}$ were respectively greater when the hit frequency was 5 , which has the $\Sigma \mathrm{A} \%, \Sigma \mathrm{E}$ and $\Sigma \mathrm{CAI}$ is $39.90 \%, 16.98$ and 6.78 respectively, indicating a higher degree of enrichment and a better distinguish ability to identify the active compounds and non-active compounds. Therefore, the identification of ICE inhibitors from Chinese herbs was carried out by the combinatorial screening method with the threshold of hit frequency was 5.

TABLEIV.COMBINATORIAL SCREENING BY 8 ICE INHIBITOR PHARMACOPHORE MODELS

\begin{tabular}{cccccc}
\hline $\mathbf{F}$ & $\boldsymbol{\Sigma} \mathbf{H a}$ & $\boldsymbol{\Sigma H t}$ & $\boldsymbol{\Sigma A} \%$ & $\boldsymbol{\Sigma} \mathbf{E}$ & $\boldsymbol{\Sigma C A I}$ \\
\hline 8 & 30 & 43 & 7.30 & 302.12 & 22.05 \\
7 & 79 & 493 & 19.22 & 69.39 & 13.34 \\
6 & 120 & 1725 & 29.20 & 30.12 & 8.80 \\
5 & 164 & 4182 & 39.90 & 16.98 & 6.78 \\
4 & 184 & 9728 & 44.77 & 8.19 & 3.67 \\
3 & 212 & 20717 & 51.58 & 4.43 & 2.29 \\
2 & 239 & 41734 & 58.15 & 2.48 & 1.44 \\
1 & 284 & 70952 & 69.10 & 1.73 & 1.20 \\
\hline
\end{tabular}

The combinatorial screening results, which were obtained by the combinatorial screening with 8 pharmacophore models, were shown in Table V. 781 compounds can fit 5 or more pharmacophore models. The Chinese herbs, which contain the hit compounds, mostly have the efficacy of 'heat-clearing and detoxifying', which is suggested in many studies to have the anti-inflammatory activity (shown in Table VI).

In summary, eight structure-based ICE inhibitor pharmacophore models were constructed by LigandScout. Combinatorial screening method by multiple pharmacophore models was proposed in this study and used to improve the efficiency of virtual screening. The hit frequency 5 was determined as the threshold to screen the active compounds from Chinese herbs. 781 molecules were obtained which were contained in Chinese herbs with 'heat-clearing and detoxifying' efficacy. The ICE inhibitory activity of hit compounds need to be further verified in vitro.

\section{ACKNOWLEDGMENT}

This work is financially supported by the Foundation of National Natural Science Foundation of China (No. 81173522) and National Key Technology R\&D Program (No. 2008BAI51B01) in Beijing University of Chinese Medicine.

TABLE V.THE HIT MOLECULES FROM CHINESE HERBS BY COMBINATORIAL SCREENING OF ICE INHIBITOR PHARMACOPHORES

\begin{tabular}{ccc}
\hline Frequency & Ht & $\boldsymbol{\Sigma} \mathbf{H t}$ \\
\hline 8 & 7 & 7 \\
7 & 84 & 91 \\
6 & 244 & 335 \\
5 & 446 & 781 \\
4 & 582 & 1363 \\
3 & 658 & 2021 \\
2 & 778 & 2799 \\
1 & 1179 & 3978 \\
\hline
\end{tabular}

TABLE VI. COMPOUNDS AND HERBS OF PREDICTED ACTIVITY AGAINST ICE

\begin{tabular}{ccc}
\hline Compounds & Species & Herb (pinyin) \\
\hline Andrographidine B & Andrographis paniculata & Chuan Xin Lian \\
Aplopaeonoside & Paeonia moutan & Mu Dan Pi \\
Cyrtophylin & Clerodendron & Lu Bian Qing \\
& cyrtophyllum. & \\
Picroside II & Picrorhiza kurrooa & Hu Huang Lian \\
Quercitrin & Hypericum perforatum & Lian Qiao \\
\hline
\end{tabular}

REFERENCES

[1] Judith M. Rollinger, Sabine Haupt, Hermann Stuppner, and Thierry Langer,Combining Ethnopharmacology and Virtual Screening for Lead Structure Discovery: COX-Inhibitors as Application Example, J. Chem. Inf. Comput. Sci.,vol. 44, 2004, pp.480-488

[2] Yili W., Steven V.O., John A.W., etc., Synthesis and evaluation of unsaturated caprolactams as interleukin-1ß converting enzyme (ICE) inhibitors, Bioorganic \& Medicinal Chemistry, vol.15, 2007, pp. 1311-1322

[3] Stephan G., Bernd L.H., Klaus K., The interleukin 1ß-converting enzyme inhibitor CrmA prevents Apl1/Fas- but not glucocorticoid-induced poly (ADP-ribose) polymerase cleavage and 
apoptosis in lymphoblastic leukemia celss, FEBS letters, vol.402, 1997, pp.36-40

[4] Osman FG., Pharmacophore perception, development, and use in drug design,1st ed.,California: InternationalUniversity Line, 2000, pp.197.

[5] Thierry L and Remy DH,Pharmacophoresand pharmacophore searchs, volume 32, Wiley-VCH, 2006, pp.338-339 